Mots. Les langages du politique

$97 \mid 2011$

Les collectivités territoriales en quête d'identité

\title{
De l'un et du divers. La région Rhône-Alpes et la mise en récit de ses langues
}

Unity and diversity. Rhône-Aples narrates its regional languages

De lo uno y lo diverso. La región Rhône-Alpes y la puesta en relato de sus lenguas

James Costa et Michel Bert

\section{OpenEdition}

\section{Journals}

Édition électronique

URL : https://journals.openedition.org/mots/20489

DOI : $10.4000 /$ mots.20489

ISBN : $15 / 11 / 2013$

ISSN : 1960-6001

Éditeur

ENS Éditions

Édition imprimée

Date de publication : 15 novembre 2011

Pagination : 45-57

ISBN : 978-2-84788-326-8

ISSN : 0243-6450

Référence électronique

James Costa et Michel Bert, " De l'un et du divers. La région Rhône-Alpes et la mise en récit de ses langues ", Mots. Les langages du politique [En ligne], 97 | 2011, mis en ligne le 15 novembre 2013, consulté le 23 avril 2022. URL : http://journals.openedition.org/mots/20489 ; DOI : https://doi.org/ $10.4000 /$ mots.20489 


\section{De l'un et du divers. La région Rhône-Alpes et la mise en récit de ses langues}

La région Rhône-Alpes constitue à première vue un exemple particulièrement emblématique de la création des régions françaises sur des critères administratifs apparemment arbitraires, imposés par le pouvoir central. Pourtant, au même titre que d'autres régions à l'identité plus marquée comme la Bretagne ou l'Alsace, le conseil régional de Rhône-Alpes a cherché à forger une image distinctive de la région (certains diront une identité), à la fois à destination des citoyens qui vivent sur son sol mais aussi dans une visée de commodification de cette image à travers des activités comme le commerce ou le tourisme. Par commodification, il faut entendre la transformation d'un objet, d'une idée, d'une construction sociale en marchandise échangeable et/ou négociable dans un marché donné (voir Heller, 2003; Duchêne, Heller, à paraître).

Notre contribution cherche à mettre en relief la manière dont les questions linguistiques ont été récemment intégrées aux autres procédés utilisés par le conseil régional de Rhône-Alpes dans sa politique régionale de création d'une image de soi à travers la mise en circulation d'un récit régional. Nous nous inscrivons de la sorte à la fois dans la continuité de travaux en analyse de discours sur la formation d'identités régionales distinctives et plaçant la région à l'interface du local et du global (voir en particulier Pearce, Wodak, 2010) ainsi que dans la suite de travaux en sociolinguistique d'Alexandre Duchêne (2008) sur la formation discursive de groupes minoritaires et majoritaires à travers la sémiotisation des questions de langue. Les travaux sur la région sont particulièrement importants à l'heure actuelle, cette unité administrative occupant une importance croissante au sein de la construction européenne comme unité d'affiliation identitaire et administrative du quotidien, et sa centralité a été soulignée par plusieurs travaux récents (Pearce, Wodak, 2010, ainsi que l'ensemble du numéro 13 [3] du European Journal of Cultural Studies, 2010 ; voir aussi Boutan et al., 2001).

Dans un premier temps, nous chercherons à comprendre comment la région met en place sa politique linguistique dans un contexte d'identité régionale

ICAR (CNRS, UMR 5191, Institut français de l'éducation, ENS de Lyon et Université de Lyon) james.costa@ens-lyon.fr DDL (CNRS, UMR 5596 et Université de Lyon)

michel.bert@univ-lyonz.fr 
peu affirmée, avant de montrer comment les langues régionales ont peu à peu fait leur entrée sur la scène politique locale. Nous analyserons enfin comment ces questions linguistiques sont mises en texte dans la construction du récit régional institutionnel officiel.

Nous nous appuierons sur certains des textes recueillis depuis la remise au conseil régional de notre rapport d'étude sur les langues de Rhône-Alpes, le franco-provençal et l'occitan (voir Bert, Costa, Martin, 2009). Depuis lors, plusieurs documents institutionnels ou para-institutionnels ont été produits : rapport remis aux élus et délibération du conseil régional lors du vote d'une délibération en faveur des langues régionales en juillet 2009, dépliant sur les langues de Rhône-Alpes destiné à être largement diffusé, textes d'appels à projets pouvant bénéficier de financements régionaux, préface du président de la région JeanJack Queyranne à un récent ouvrage sur la littérature franco-provençale et occitane (Martin, Rixte, 2010). Le site internet du conseil régional diffuse par ailleurs des informations sur les langues régionales en mettant plusieurs documents à disposition du public. Les extraits de textes analysés dans cet article proviennent de ce site ainsi que du texte de la délibération; ils seront analysés d'un point de vue critique, de manière à faire apparaître les enjeux idéologiques sous-jacents.

Les questions de positionnement identitaire sont au cœur du discours régional. Le thème de l'identité régionale étant néanmoins trop vaste pour être traité dans cet article, il nous semble pertinent d'analyser nos données en termes de ce que Martina Avanza et Gilles Laferté (2005) désignent par identification. Partant du principe que la notion d'identité était trop imprécise pour être opératoire en sciences sociales, et s'appuyant notamment sur les travaux de Rogers Brubaker (2001), ils décomposent celle-ci en trois aspects complémentaires : identification, image sociale et appartenance. La notion d'identification est ainsi définie :

[...] l'identification pourrait qualifier toute action sociale où l'attribution identitaire est extérieure, s'exerçant sur un individu, dans le cadre d'une institution sociale, selon une technique codifiée. (Avanza, Laferté, 2005, p. 142)

La notion d'identification recouvre ainsi les processus extérieurs aux individus, mécanismes conscients par lesquels une institution va chercher à produire une identité pour une catégorie d'individus (les Rhônalpins) et un espace (RhôneAlpes). Ce qui est en jeu dans les documents analysés ici, c'est donc, plus que l'identité régionale, la manière dont l'institution cherche à identifier un territoire particulier, en fonction d’intérêts localement situés.

\section{Rhône-Alpes : naturaliser une construction administrative}

Si la région Rhône-Alpes telle que nous la connaissons actuellement est une construction politique et économique datant des années soixante, elle 
n'échappe pas à une tentative de naturalisation qui est à la fois la marque de la fondation des États-nations modernes (Bauman, Briggs, 2003) et des régions européennes contemporaines (Pearce, Wodak, 2010; Felgenhauer, 2010).

\section{L'invention d'une histoire}

Il est à ce titre significatif que le site internet du conseil régional présente la création de Rhône-Alpes non pas comme une contingence historique liée à des intérêts économiques situés mais comme le parachèvement logique d'une histoire millénaire :

[Extrait 1]

La soierie lancée par François ler (1536), relancée par Napoléon au milieu du $\mathrm{XIX}$ siècle, essaime à travers tous les départements et crée la région avant qu'elle n'existe. La création de l'entité administrative un siècle plus tard (au début des années 1960) consacre cette cohérence en créant un ensemble régional regroupant les trois agglomérations qui en déterminent la puissance : Lyon, Saint-Étienne, Grenoble. ${ }^{1}$

Ce paragraphe qui consacre l'existence de la région non seulement comme entité administrative, mais aussi comme unité naturelle, est particulièrement éloquent. Il mentionne, d'une part, les fondateurs de la centralisation française, François Ier et Napoléon, et d'autre part, il renvoie à la naissance de la région à l'époque contemporaine, mais en nominalisant ${ }^{2}$ le processus ("création »), ce qui permet d'en exclure les acteurs principaux - c'est-à-dire le pouvoir politique central de l'époque - et d'en renforcer l'immanence. L'idée de «création », qui revient trois fois dans le paragraphe, est au cœur du discours officiel régional et fonctionne comme un pendant immanent à l'idée de «lancement», associée quant à elle à des personnages historiques. La «création » permet dans le discours d'associer l'action humaine à l'existant (les villes, le paysage) et de naturaliser Rhône-Alpes comme un ensemble cohérent.

En ce sens, l'entreprise régionale s'inscrit dans un processus de lutte des classements et entre en concurrence avec d'autres centres d'identification potentiels des citoyens de la région : départements, communes, entités régionales historiques (on pensera en particulier à la Savoie ou à la Bresse) ou touristiques (comme la «Drôme provençale »), voire d'espaces linguistiques plus larges, par exemple celui de l'occitan, qui englobe le tiers sud de la France actuelle. La volonté de naturaliser la région s’apparente en ce sens à ce que

1. Site de la région Rhône-Alpes [http://www.rhonealpes.fr/57-histoire-rhone-alpes.htm], section «Histoire». La validité de l'ensemble des liens donnés dans cet article a été vérifiée en juin 2011.

2. Les processus de nominalisation sont définis par Fairclough (1992, p. 27) comme la conversion d'une clause en nom, action grammaticale permettant de ne pas nommer le/les sujet/s de l'action ainsi décrite. 
Pierre Bourdieu $(1980,2001)$ nomme un «acte de magie sociale », acte qui tente de «produire à l'existence la chose nommée» (2001, p. 286). Bourdieu précise cependant qu'un tel acte «peut réussir si celui qui l'accomplit est capable de faire reconnaître à sa parole le pouvoir qu'elle s'arroge par une usurpation provisoire ou définitive » (ibid.). La question centrale est donc une question d'autorité et de reconnaissance de cette autorité. Autrement dit, l'enjeu en termes d'identité pour le conseil régional est de parvenir à transformer un acte d'identification en un sentiment d'appartenance de la part des habitants du territoire. Là où certaines régions bénéficient d'un avantage de départ comme la région Bretagne ou l'Alsace, en particulier du fait de la permanence d'un toponyme historiquement marqué, Rhône-Alpes se trouve dans une posture délicate qui oblige l'instance dépositaire de son nom à employer des stratégies de naturalisation différentes. L'histoire peut servir le dessein régional, mais au prix d'un gommage de l'agentivité des décideurs politiques des années soixante qui ont fait exister la région en tant qu'entité administrative française, au profit de Grands ancêtres plus illustres, qui sont pourtant paradoxalement associés au développement du centralisme étatique français.

L'histoire véhicule d'autres enjeux qui dépassent le seul moment de «création » de la région. Ce moment est en effet présenté, toujours sur le site du conseil régional, comme l'aboutissement d'un temps plus long :

[Extrait 2]

Plus de 30000 ans avant notre ère : les occupants de la grotte Chauvet (Ardèche) dessinent les premières œuvres d'art connues de l'humanité.

Avant l'arrivée des Romains, pas moins de 8 peuples gaulois se partagent le territoire de la future région Rhône-Alpes : les Helviens du côté méridional, les Allobroges en Dauphiné, les Ceutrons en Tarentaise, les Médulles en Maurienne, les Ambarres dans l'Ain, les Ségusiaves autour de Lyon et de Roanne et, de manière plus limitée, les Cavares et les Voconces installés surtout au sud de la région. 3

Ce paragraphe précède immédiatement celui analysé plus haut. Il annonce de manière téléologique l'aboutissement de l'Histoire que représentera la «création » de la région Rhône-Alpes. La référence à la grotte Chauvet permet, outre l'enracinement dans une histoire plurimillénaire, de faire le lien avec le présent, cette grotte ayant été découverte en 1994, à une époque où Rhône-Alpes existait déjà donc. Cette continuité avec le passé est également apparente dans le choix d'identifier et de nommer les huit tribus gauloises ayant occupé la région, pendant historique aux huit départements actuels qu'elle comprend. Les Cavares et les Voconces préfigurent en quelque sorte, de par leur marginalité sur l'espace régional et leurs associations avec d'autres régions plus méridionales, les départements actuels occitanophones de Drôme et d’Ardèche.

3. Site de la région Rhône-Alpes [http://www.rhonealpes.fr/57-histoire-rhone-alpes.htm], section «Histoire». 
Cette histoire régionale volontairement simplifiée, et faisant appel à une iconographie riche et largement partagée (la grotte Chauvet, les Gaulois, François Ier, Napoléon) est destinée à diffuser un récit régional cohérent et assurant une continuité diachronique. Il est intéressant de noter que ce récit est repris presque mot pour mot par l'entrée «Rhône-Alpes» de l'encyclopédie en ligne Wikipédia4, signe de la diffusion du récit régional institutionnel.

\section{Tensions entre l'un et le multiple}

La dimension historique est intégrée à un tout qui, dans la section «Découvrir Rhône-Alpes » du site du conseil régional, englobe des éléments tels que la géographie régionale, l'économie, le patrimoine, l'art de vivre, le tourisme et une «carte d’identité » synthétique de la région. Si, dans la partie historique, l'accent est mis sur une continuité temporelle, cette présentation s'inscrit explicitement dans une tentative de réconcilier l'un et le multiple, l'unité régionale et la diversité de ses manifestations culturelles, humaines et paysagères.

En ce sens, la construction d'une identité régionale comme celle de RhôneAlpes s'inscrit en tension entre les tendances idéologiques unitaires des Étatsnations modernes et d'autres influences idéologiques mettant l'accent sur la valorisation de la diversité, par exemple celles nées au lendemain de la seconde guerre mondiale et portées par l'Union européenne et surtout par le Conseil de l'Europe.

Cette tension s'exprime à travers l'usage récurrent de termes comme contraste ou diversité, et se retrouve à travers l'ensemble du site. Par exemple, dans la section « géographie » du site internet du conseil régional, on peut lire:

[Extrait 3]

L'art de la diversité5

Les glaciers au Nord-est [sic], les lavandes au Sud... une fois de plus Rhône-Alpes prouve qu'elle est terre de contrastes.

Il existe néanmoins un dénominateur commun entre les différents territoires qui composent la région : le Rhône. Le «fleuve-roi» borde ou traverse en effet les huit départements de la Région. ${ }^{6}$ [Guillemets dans l'original]

L'indication « une fois de plus » permet d'insister sur la récurrence du rappel de la diversité dans la communication de l'institution régionale. On trouve ce principe clairement affirmé également dans la section «carte d’identité » du site :

4. Site Wikipedia [http://fr.wikipedia.org/wiki/Rhône-Alpes], entrée «Histoire».

5. Il s'agit d'un titre. La phrase suivante forme un chapeau et apparaît en gras dans le texte original.

6. Site de la région Rhône-Alpes [http://www.rhonealpes.fr/56-geographie-rhone-alpes.htm], section «Géographie». 
[Extrait 4]

Rhône-Alpes tire son unité de ses mille et une facettes géographiques et climatiques. Mais ce sont avant tout ses habitants qui font chaque jour de Rhône-Alpes une région dynamique, attractive où il fait bon vivre. [...]

La région Rhône-Alpes est placée au carrefour naturel de grands axes de communication nationaux et européens. Cette situation en fait une Région ou la diversité est le maître mot. Ce puzzle à la fois géographique, climatique, sociologique et culturel pourrait être un handicap. Rhône-Alpes en a fait sa force.7 [Nous soulignons]

La tension entre unité et diversité reste immanente, à travers la métaphore du «puzzle» associée à celle du «handicap», menace omniprésente renversée symboliquement par le conseil régional lui-même, désigné par le nom de la région elle-même, Rhône-Alpes. Le territoire et l'institution en viennent ainsi à se confondre. Par un procédé de répercussion à d'autres niveaux, les caractéristiques de la géographie régionale se trouvent reflétées dans la composition même de la population régionale. Peu à peu, l'image de la multiplicité se répercute de domaine en domaine. C'est donc dans ce contexte idéologique particulier que le discours régional sur les questions linguistiques se doit de trouver une place.

\section{Rhône-Alpes en Europe}

Un dernier élément nous semble devoir être mentionné pour comprendre le contexte idéologique dans lequel le conseil régional de Rhône-Alpes a développé un discours sur les langues régionales parlées sur le territoire. Il concerne la manière dont la région est positionnée discursivement par rapport à d'autres régions européennes. Cette comparaison permet de mettre en avant certains éléments clefs de l’identification de la région.

La région fait partie de deux ensembles transrégionaux : les «Quatre moteurs de l'Europe », d'une part, et l'Eurorégion Alpes-Méditerranée, d'autre part.

Parmi les «Quatre moteurs», on trouve, en plus de Rhône-Alpes, la Catalogne, la Lombardie et le Bade-Wurtemberg. L'Eurorégion comprend quant à elle, outre Rhône-Alpes, la région Provence - Alpes - Côte-d'Azur en France, et le Piémont, le Val d'Aoste et la Ligurie en Italie. Ces régions, dont certaines parmi les plus dynamiques économiquement en Europe, sont toutes identifiées par un toponyme historiquement marqué. Outre une fonction dénotative, à ces toponymes est ainsi associée une dimension connotative importante, qui génère à son tour un «investissement représentationnel-ethnosocioculturel » (Boyer, 2008, p.10; Boyer, 2003) susceptible de convoquer un certain nombre d’images fortes, dont ne peut se prévaloir la région Rhône-Alpes.

7. Site de la région Rhône-Alpes [http://www.rhonealpes.fr/23-dynamisme-en-rhone-alpes.htm], section «Carte d’identité». 
Les partenariats économiques et culturels avec ces régions sont donc susceptibles d'influer sur la politique d'identification du conseil régional, au moins en termes de commodification de l'identité régionale à des fins commerciales. Pour certaines de ces régions européennes, les questions linguistiques jouent un rôle considérable dans la définition de l'identification régionale, mais aussi dans la construction d'une appartenance à un ensemble politique. On pensera bien sûr à la Catalogne et au Val d'Aoste, mais aussi, dans une moindre mesure, au Piémont ou à la Provence.

C'est donc dans ce cadre à la fois politique et économique qu'il faut comprendre la recherche d'un positionnement particulier face aux questions linguistiques. À un cadre français traditionnellement rigide et centralisé, imposant un toponyme fondé sur la seule géographie physique et des limites basées sur des critères économiques, correspond une volonté d'intégration européenne qui impulse une dynamique idéologique valorisant le divers. Le processus d'identification de la région Rhône-Alpes repose donc ici aussi sur la tension entre ces deux pôles. Le défi principal de l'institution régionale consiste de fait à diffuser une identification suffisamment forte pour influencer la construction d'une image sociale apte à s'imposer, et surtout capable d'influer sur le sentiment d'appartenance des habitants de la région.

\section{De l'absence de langue rhônalpine à la valorisation de deux langues}

C'est par une approche de type positiviste que le conseil régional a souhaité appréhender la question des langues rhônalpines. En effet, à la suite de plusieurs rencontres avec des militants du franco-provençal et de l'occitan, et sur l’impulsion de plusieurs élus, les services culturels régionaux rédigèrent un appel d'offres public indiquant la volonté de l'institution régionale de mieux connaître la réalité des pratiques linguistiques locales. Le marché fut emporté par une association de laboratoires scientifiques rhônalpins, et l'enquête qui s'ensuivit donna finalement lieu à un vote au conseil régional en juillet 2009, ouvrant ainsi la voie à la mise en place d'une politique linguistique régionale.

\section{L'enquête Fora}

C'est dans ce contexte que fut lancée, en 2007, l'enquête intitulée «Francoprovençal et occitan en Rhône-Alpes » (Fora), pilotée par l'Institut Gardette de l'Université catholique de Lyon en partenariat avec divers laboratoires lyonnais (ICAR, DDL) et grenoblois (Centre de dialectologie). Le cas rhônalpin est atypique : en effet, en Bretagne, en Aquitaine, et plus récemment en MidiPyrénées, les enquêtes de ce type ont été confiées à des cabinets d'expertise 
et non à des laboratoires universitaires. En conséquence, l'enquête Fora (Bert et al., 2009) est davantage le résultat d'enquêtes qualitatives et d'une connaissance fine des terrains étudiés que d'enquêtes quantitatives, comme c'est le cas dans les autres régions françaises.

Par ailleurs, l'aspect évaluatif de l'enquête ne doit pas masquer la nature performative de la demande initiale. L'appel d'offres stipulait en effet la condition suivante: «En préambule, il apparaît important de nommer et de définir les langues de Rhône-Alpes» (cité dans Bert et al., 2009, p. 12). La demande institutionnelle impliquait ainsi de faire des choix à la fois en termes de limites linguistiques et de noms de langues ${ }^{8}$. Malgré sa nature positiviste, la commande institutionnelle était donc par certains aspects un acte de magie sociale cherchant à produire des objets destinés à être consacrés officiellement.

L'enquête Fora visait, d'une part, à produire une connaissance sur les pratiques langagières en franco-provençal et en occitan dans la région et, d'autre part, à fournir des scénarios possibles de mise en place d'un plan de politique linguistique régionale. Le conseil régional souhaitait donc s'engager dans une forme de valorisation d'un patrimoine linguistique mais aussi, dans une certaine mesure, dans la mise en œuvre d'un plan de renversement de la substitution linguistique, pour utiliser la terminologie employée par Joshua Fishman (1991)9. Dans la préface à une récente anthologie des littératures rhônalpines en franco-provençal et en occitan (Martin, Rixte, 2010, p. 6), Jean-Jack Queyranne, le président de la région Rhône-Alpes, évoque même la «reconquête» des langues régionales.

\section{La réception de l'enquête Fora}

Le rapport d'enquête fut remis au conseil régional en mai 2009 , et en juillet de la même année, une proposition de délibération fut soumise aux élus régionaux en vue de l'adoption de mesures destinées à sauvegarder les langues régionales de Rhône-Alpes. Le projet de délibération, intitulé « Reconnaître, valoriser, promouvoir l'occitan et le franco-provençal, langues régionales de RhôneAlpes», fut adopté par une majorité de conseillers régionaux ${ }^{10}$.

La principale motivation exprimée par le texte de la délibération renvoie à l'incarnation locale d'enjeux globaux. Ainsi, le texte s'ouvre sur le rappel de

8. Il faut signaler ici qu'il existe en domaine d'oc un violent débat idéologique entre partisans d'un «occitan » dont le provençal serait une variante dialectale, et tenants du provençal comme langue autonome (voir Blanchet, 2002, ou Costa, 2010). En franco-provençal, les tensions sont moins vives, mais il existe cependant des débats quant à l'utilisation de franco-provençal, arpitan, ou de désignants plus locaux : savoyard, bressan, etc. (voir Costa, 2011).

9. Pour une description détaillée de l'enquête, voir Bert et al., 2009 et Bert, 2011.

10. Le texte de la délibération et du rapport l'accompagnant, dont sont tirés les extraits 5 et 6 , est consultable sur le site de la Région Rhône-Alpes [http://www.rhonealpes.fr/485-languesregionales-rhone-alpes.htm\#par10082]. 
la défense de la diversité culturelle comme une des priorités de l'action régionale. Les deux paragraphes suivants renforcent ce lien entre enjeux globaux et locaux :

\section{[Extrait 5]}

L'émergence du fait régional; la prise de conscience mondiale du danger d'extinction qui pèse sur de très nombreuses langues, et en particulier sur les langues minoritaires et régionales; les mesures prises par nombre de pays européens en faveur de leur diversité linguistique interne; la reconnaissance par la Constitution de l'appartenance de ces langues « au patrimoine de la France»; la création, au mois d'octobre 2007, de l'Eurorégion Alpes-Méditerranée, dont tous les membres à l'exception de la Ligurie ont en partage l'une des langues régionales parlées en Rhône-Alpes (l'occitan en PACA, le franco-provençal en Val d'Aoste, les deux langues au Piémont) : autant d'éléments qui rendaient indispensable la prise en compte de ce patrimoine immatériel, constitutif de l'identité rhônalpine et cependant très menacé.

Parmi les 6000 à 10000 langues parlées aujourd'hui dans le monde, la très grande majorité est menacée d'extinction. Les linguistes estiment qu'une langue s'effacerait ainsi tous les 15 jours! [...] Une langue est une vision du monde originale, porteuse de valeurs, de culture et d'identité. Et elle n'existe pas en dehors des personnes qui choisissent de l'utiliser. ${ }^{11}$

Le discours régional transfère le discours global sur les «langues en danger» au niveau régional, en le liant avec les niveaux politiques européens (référence aux politiques d'autres pays) et français (référence à la modification de la Constitution en juillet 2008). Le texte vise à créer un contexte discursif présentant comme inévitable l'adoption de la délibération par les élus, en se plaçant au niveau de l'analogie entre les menaces pesant sur la diversité linguistique et sur la biodiversité. L'arrière-plan idéologique se réfère ici aux discours écologistes sur les langues, qui lient ces divers types de menaces (voir par exemple Nettle, Romaine, 2003). Or, comme le fait remarquer Deborah Cameron (2007), ce type de discours, centré sur les langues et non sur les acteurs sociaux, permet d'occulter les rapports de pouvoir et les enjeux locaux liés aux mouvements de revitalisation linguistique, notamment en termes de répartition du pouvoir matériel et, surtout, symbolique. Le texte du conseil régional est également centré sur des entités abstraites, les langues de Rhône-Alpes et, au cours des paragraphes introductifs, ne mentionne les locuteurs que dans une seule phrase, la dernière dans l'extrait de texte donné ci-dessus : « [e]t elle n'existe pas en dehors des personnes qui choisissent de l'utiliser ». Les utilisateurs de ces langues restent donc pour le moins marginaux dans le discours. Les pratiques de langues régionales sont par ailleurs marquées par l'usage du verbe «choisir», indiquant par là même leur nature optionnelle. En d'autres

11. Rapport n 49.11450 du conseil régional de Rhône-Alpes soumis à délibération en juillet 2009 , p. 1 , paragraphes 2 et 3 . 
termes, la défense des langues régionales passe par un effacement des locuteurs, ce qui peut se voir comme une tentative de dépolitiser la question : le sauvetage des langues régionales est présenté comme une question de respect de la diversité prise comme une abstraction, et non comme la prise en compte d'une question toujours éludée en France, celle de pratiques langagières mettant en cause le dogme de l'homogénéité (Blommaert, Verschueren, 1998), fondement idéologique de l'État-nation français.

Outre ces considérations, qui ancrent le discours régional dans la tension entre les pôles idéologiques décrits plus haut, il est intéressant de noter quels éléments du rapport Fora susceptibles d'intéresser les élus régionaux ont été retenus par les rédacteurs de la délibération. Le passage suivant s’intéresse plus particulièrement au rôle éventuel que pourraient jouer les questions linguistiques dans la définition de la politique d’identification de la région :

[Extrait 6]

Faisant observer que le dialecte occitan le plus communément parlé en RhôneAlpes en dehors du sud de l'Ardèche et de la Drôme, le vivaro-alpin, ou nord-occitan de l'Est, comporte de nombreux traits communs avec le franco-provençal, les auteurs l'expliquent par le fait que «la région Rhône-Alpes a été constituée autour de Lyon, pôle économique, et que le franco-provençal tire son origine de l'influence linguistique exercée par Lugdunum» et en concluent que «la langue vernaculaire (...) constitue l'élément le plus fort et le plus ancien de l'identité rhônalpine, car elle prouve qu'il a existé pendant près de deux millénaires une communauté linguistique entre tous les petits pays qui forment cette région $» .{ }^{12}$

Le texte présenté en italiques dans l'extrait provient de l'introduction au rapport Fora, rédigée par Jean-Baptiste Martin et qui visait à définir les langues de Rhône-Alpes. Ce passage vient ici alimenter le discours institutionnel de production d'une identification servant à légitimer l'existence de la région, au même titre que le travail de la soie, comme nous l'avons vu précédemment. La même tension entre l'un et le multiple observée dans la présentation des dimensions géographiques, climatiques et culturelles de la région est également à l'œuvre ici. Au-delà de la présence de deux langues traditionnellement identifiées comme distinctes dans les études romanes traditionnelles, l'influence de Lyon permet d'établir un fondement antique à l'existence de RhôneAlpes en minimisant les différences et en permettant de poser une unité linguistique, et, partant, historique.

Un premier résultat de l'utilisation des questions linguistiques par le conseil régional tient donc dans la possibilité d'une normalisation de l'entité régionale rhônalpine : malgré un toponyme récent et ne faisant pas référence aux territoires historiques ainsi englobés, malgré une diversité économique,

12. Ibid., p.3-4, italiques dans le texte original - elles marquent une citation du rapport Fora (Bert etal., 2009). 
culturelle, géographique et climatique, il existerait des pratiques linguistiques susceptibles de se rapporter à la région Rhône-Alpes, ce qui, potentiellement, peut placer la région au rang des régions françaises à l'identité plus assurée. Surtout, dans la mise en texte présentée dans l'extrait 6, les désignants occitan et franco-provençal disparaissent progressivement au profit d'une seule «langue vernaculaire». Présentées ainsi, les pratiques linguistiques rhônalpines peuvent être dissociées des ensembles plus larges auxquels les termes occitan et franco-provençal renvoient habituellement, et se trouver associées plus étroitement au territoire régional.

\section{La « langue vernaculaire » dans le récit régional}

Compte tenu du niveau actuel des pratiques, les langues régionales ne peuvent, en Rhône-Alpes, fonder seules le récit régional. L'originalité du récit rhônalpin consiste à rester suffisamment large pour permettre d'accueillir la problématique linguistique et de l'y intégrer pour renforcer un discours préexistant. Si les langues régionales sont réifiées dans le discours institutionnel, deux enjeux semblent présents : d'une part, l'intégration de l'histoire linguistique comme élément de consolidation de la légitimité de l'entité territoriale régionale et de son exécutif politique; d'autre part, l'intégration de ces langues dans une stratégie de promotion d'une image sociale de la région, en particulier à travers le tourisme. Les langues, ainsi commodifiables - c'est-à-dire, rappelonsle, qu'une valeur intrinsèque leur est attribuée et qu'elles sont valorisables sur un marché donné-, peuvent ainsi intégrer le discours officiel, aux côtés du développement durable, par exemple, qu'elles peuvent habilement soutenir.

Les langues régionales de Rhône-Alpes sont tout à la fois suffisamment intéressantes pour contribuer à la naturalisation de l'idée d'une région RhôneAlpes tout en restant assez lointaines pour n'y contribuer que marginalement, sans empiéter sur ce qui forme la base de la stratégie d'identification de la région: l'innovation, le développement d'une industrie de pointe, le tourisme, en particulier de sports d'hiver. Par rapport à la soierie, l'élément linguistique peut être considéré comme plus suggestif dans une région où une autre langue, le français, constitue un des piliers, sinon le pilier principal, de l'État-nation dans lequel elle est incluse.

Le contexte européen, à la fois à travers les «Quatre moteurs » et à travers la constitution de l'Eurorégion, fait par ailleurs naître des besoins nouveaux en termes d'identification, auxquels la présence et la valorisation d'une langue peuvent répondre au moins partiellement.

L'enjeu principal pour le conseil régional reste cependant la production d'éléments discursifs de référence auxquels les habitants puissent s'identifier, dans le but pour l'institution de perdurer en tant que telle et d'imposer 
ses catégorisations du réel. La stratégie adoptée par le conseil régional semble reposer plus sur la valorisation des langues en elles-mêmes, comme symboles, que sur des orientations qui viseraient à s'intéresser aux enjeux sociaux auxquels sont confrontées les populations, ou les individus, parlant (encore) une langue régionale. La stratégie porte donc sur la langue et non sur les individus : en ce sens, malgré une apparente importance accordée à la diversité, le discours rhônalpin s'intègre dans la suite des discours traditionnels sur les «langues menacées » (voir Duchêne, 2008, p. 6-8), qui négligent les dynamiques sociales dans lesquelles sont impliqués les locuteurs, mais aussi les promoteurs, de ces langues.

\section{Références}

Avanza Martina, LAferté Gilles, 2005, «Dépasser la “construction des identités”? Identification, image sociale, appartenance », Genèses, nº 61 (4), p. 134-152.

BAUMAn Richard, BRIGgS Charles L., 2003, Voices of Modernity. Language Ideologies and the Politics of Inequality, Cambridge, Cambridge University Press.

BERT Michel, 2011, «Situation sociolinguistique du franco-provençal. L'étude FORA», Langues et Cité, nº 18, p. 5.

Bert Michel, Costa James, MARTIN Jean-Baptiste, 2009, Étude FORA. Franco-provençal et occitan en Rhône-Alpes, Lyon, Institut Pierre Gardette, INRP, ICAR, DDL, en ligne [http:// icar.univ-lyon2.fr/projets/ledra/documents/Etude_FORA_rapport_d\% $3 \%$ Agfinitif. pdf]. Consulté le 15 juin 2011.

BLANCHET Philippe, 2002, Langues, cultures et identités régionales en Provence. La métaphore de l'aïoli, Paris, L'Harmattan.

BLommaert Jan, Verschueren Jef, 1998, «The role of language in European nationalist ideologies", Language Ideologies. Practice and Theory, B. Schieffelin, K. A. Woolard, P.V. Kroskrity éd., Oxford, Oxford University Press, p. 189-210.

BOURDIEU Pierre, 1980, "L'identité et la représentation. Éléments pour une réflexion critique sur l'idée de région », Actes de la recherche en sciences sociales, n 35 (1), p. 63-72.

Bourdieu Pierre, 2001, Langage et pouvoir symbolique, Paris, Fayard.

BoutAn Pierre, MARTEL Philippe, Roques Georges éd., 2001, Enseigner la région, Paris, L'Harmattan.

BOYER Henri, 2003, De l'autre côté du discours, Paris, L'Harmattan.

- 2008, "Fonctionnements sociolinguistiques de la dénomination toponymique», Mots. Les langages du politique, $\mathrm{n}^{\circ} 86$, Toponymes. Instruments et enjeux, p. 8-21.

BRUBAKER Rogers, 2001, "Au-delà de l'“identité" ", Actes de la recherche en sciences sociales, no139 (4), p. 66-85.

CAMERON Deborah, 2007, «Language endangerment and verbal hygiene. History, morality and politics », Discourses of Endangerment, A. Duchêne, M. Heller éd., Londres, New York, Continuum, p. 268-285. 
COSTA James, 2010, «Revitalisation linguistique. Discours, mythe et idéologie. Approche critique de mouvements de revitalisation en Provence et en Écosse», thèse de doctorat soutenue sous la direction de Marinette Matthey, Université de Grenoble.

- 2011, «Patois, gaga, savoyard, franco-provençal, arpitan. Quel nom pour une langue?», Langues et Cité, $\mathrm{n}^{0} 18, \mathrm{p} .6$.

Duchêne Alexandre, 2008, Ideologies across Nations. The Construction of Linguistic Minorities at the United Nations, Berlin, New York, Mouton de Gruyter.

DuChêne Alexandre, Heller Monica éd., à paraître, Language in Late Capitalism. Pride and Profit, Londres, New York, Routledge.

FaIRCLOUgh Norman, 1992, Discourse and Social Change, Cambridge, Polity.

Felgenhauer Tilo, 2010, "Regional identity as celebration and routine. "Mitteldeutschland's" glorification and its taken-for-granted meaning in media content », European Journal of Cultural Studies, $n^{0} 13$ (1), p. 63-80.

FISHMAN Joshua A., 1991, Reversing Language Shift. Theoretical and Empirical Foundations of Assistance to Threatened Languages, Clevedon, Philadelphie, Multilingual Matters.

Heller Monica, 2003, "Globalization, the new economy, and the commodification of language and identity », Journal of Sociolinguistics, $n^{\circ} 7$ (4), p. 473-492.

MARTIN Jean-Baptiste, RIXTE Jean-Claude, 2010, Huit siècles de littérature francoprovençale et occitane en Rhône-Alpes, Lyon, Livres EMCC.

Nettle Daniel, Romaine Suzanne, 2003, Ces langues, ces voix qui s'effacent, Paris, Autrement.

PeARCE Lynne, Wodak Ruth, 2010, " (Re) constructing the region in the 21st century», European Journal of Cultural Studies, no 13 (1), p. 3-8. 